\title{
An autopsy case of pulmonary fissure induced by zygomycosis
}

This article was published in the following Dove Press journal:

International Journal of General Medicine

10 July 2013

Number of times this article has been viewed

\author{
Yuichiro Imai' \\ Yasushi Adachi ${ }^{2,3}$ \\ Takashi Kimura ${ }^{4}$ \\ Chikara Nakano 5 \\ Toshiki Shimizu ${ }^{4}$ \\ Ming Shi \\ Mitsuhiko Okigaki ${ }^{6}$ \\ Tomohiko Shimo' \\ Kazunari Kaneko' \\ Susumu Ikehara ${ }^{2}$ \\ 'Department of Pediatrics, \\ Kansai Medical University, Osaka, \\ ${ }^{2}$ Department of Stem Cell Disorders, \\ Kansai Medical University, Osaka, \\ ${ }^{3}$ Division of Clinical Pathology, \\ Toyooka Hospital, Hyogo, ${ }^{4}$ First \\ Department of Internal Medicine, \\ Kansai Medical University, Osaka, \\ ${ }^{5}$ Second Department of Internal \\ Medicine, Kansai Medical University, \\ Osaka, 'Department of Cardiovascular \\ Medicine, Kyoto Prefectural University \\ School of Medicine, Kyoto, Japan
}

Correspondence: Susumu Ikehara Department of Stem Cell Disorders, Kansai Medical University, 10-15 Fumizono-cho, Moriguchi City, Japan 570-8506

Tel +8 I 66992 I00I ext 2470

Fax +8I 669948283

Email ikehara@takii.kmu.ac.jp

\begin{abstract}
For immunodeficient patients, fungi are life-threatening pathogens. In this paper, we present an autopsy case of combined zygomycosis and aspergillosis. A female in her 70s on chronic hemodialysis was admitted to a hospital suffering bloody sputum, dyspnea, and fever, probably due to perinuclear anti-neutrophil cytoplasmic antibody-related vasculitis. Antibiotics were administered and immunosuppressive therapy was started, resulting in an improvement in her condition. Pneumonia later developed, followed by pulmonary bleeding and intractable pneumothorax from which she ultimately died. On autopsy, the upper lobe of the left lung was found to have hemorrhagic necrosis and showed a large longitudinal fissure. Microscopically, Zygomycota were observed in both the lungs and heart, while Aspergillus was found in the middle lobe of the right lung. Zygomycosis, which usually has a poor prognosis, is assumed to have induced hemorrhagic infarction of the lungs, inducing pulmonary bleeding and necrosis, despite the use of lipid formulations of amphotericin B, which are effective medicines against Zygomycota.
\end{abstract}

Keywords: pulmonary fissure, zygomycosis, aspergillosis, lung, immunosuppression

\section{Introduction}

Fungi are responsible for life-threatening infections in patients with immunodeficiency. ${ }^{1}$ Common pathogens are Candida and Aspergillus. ${ }^{2}$ However, there has been an increase in the number of patients with zygomycosis. This is probably due to the increased use of immunosuppressive therapies and newly developed antifungal agents, which are effective against Candida and Aspergillus but not against Zygomycota. Thus, cases have been identified as being associated with leukemia, ${ }^{3}$ aplastic anemia, ${ }^{4}$ bone marrow transplantation, ${ }^{3}$ diabetes mellitus, ${ }^{5}$ renal disease,${ }^{6}$ burns, ${ }^{7}$ and corticosteroid therapy. ${ }^{3,7}$ The most common clinical zygomycosis manifestations are rhino-orbital-cerebral, cutaneous, pulmonary, disseminated, and gastrointestinal. ${ }^{8}$ The most clinically important Zygomycota are those of the Mucorales order, which are widespread in nature. The likelihood of infection following ingestion or inhalation depends on the host's resistance mechanisms rather than on the number of infectious particles. ${ }^{8}$ Pulmonary zygomycosis has been reported in immunodeficient patients, with extremely poor prognosis. ${ }^{9}$

In this paper, we present an autopsy case of combined zygomycosis and aspergillosis associated with immunosuppressive therapy.

\section{Case report}

A female patient in her $70 \mathrm{~s}$ on chronic hemodialysis due to chronic renal failure presented with bloody sputum, dyspnea, and fever. This was the first episode of these 
symptoms she had experienced. As her condition worsened, she was admitted to hospital 3 days after the onset of the disease. She was diagnosed with pulmonary bleeding, probably due to perinuclear anti-neutrophil cytoplasmic antibody (p-ANCA)-related vasculitis, as she was positive for $\mathrm{p}-\mathrm{ANCA}$ $(138 \mathrm{U} / \mathrm{mL}$ [normal $<9.0 \mathrm{U} / \mathrm{mL}])$. Consolidation of the upper lobes in the bilateral lungs was found in her chest X-ray and computed tomography image (Figure 1). Cultures of sputum and blood were negative. Plasmapheresis, steroid pulse, and administration of azathioprine and antibiotics (meropenem, sulfamethoxazole/trimethoprim, and fluconazole) were carried out. Her dyspnea improved and C-reactive protein (CRP), which was $7.7 \mathrm{mg} / \mathrm{dL}$ on admission, gradually decreased to $2.2 \mathrm{mg} / \mathrm{dL}$ by 2 weeks following admission. Consolidation areas of the lungs also decreased (Figure 1).

Subsequently, the pulmonary bleeding reappeared and dyspnea developed, probably due to pneumonia. Pneumothorax occurred (Figure 1) and she needed mechanical ventilation. Three weeks after admission, she developed pneumonia (Figure 1) and the CRP titer increased again. Candida species were detected on blood culture at 21 days after admission. Micafungin was started, replacing the fluconazole. Twenty-five days after admission, levofloxacin and lipid formulations of amphotericin B (AMB) were also started.

However, her dyspnea continued to develop and she died 4 weeks following her admission. Beta-D-glucan was negative in her serum until 21 days after her admission, but increased to $35.2 \mathrm{pg} / \mathrm{mL}$ at 26 days after her admission. p-ANCA gradually decreased during her hospitalization until it finally reached the normal range 2 days before her death.

\section{Pathology report}

Four hours after her death, an autopsy was carried out. There were some purpuras on the skin.

\section{Macroscopic examination}

Both lungs were found to show congestion and edema; the weights of the left and right lung were $550 \mathrm{~g}$ and $640 \mathrm{~g}$, respectively (Figure 2). Most of the upper lobe of the left lung displayed hemorrhagic necrosis and there was a $9 \mathrm{~cm}$ long longitudinal fissure in the lobe. Cavities were found in the middle lobe of the right lung. Bloody pleural effusion in bilateral thoracic cavities and thickening of pleura in the left lung were observed. There was no significant disorder in the heart. The weight of the left kidney was $15 \mathrm{~g}$; this was assumed the result of hypoplasia. The weight of the right kidney was $48 \mathrm{~g}$. The kidney cortex was thin $-1 \mathrm{~mm}$ in the left kidney and $2 \mathrm{~mm}$ in the right.

\section{Microscopic examination}

Massive bleeding in most alveoli and a large necrotic area were observed in the upper lobe of the left lung. Non-septate irregularly branched hyphae of Zygomycota existed in the bilateral lungs and heart (Figures 3 and 4). The hyphae were frequently observed around and within the blood vessels, causing obstruction and thromboembolization of blood vessels, which turned into hemorrhagic infarction
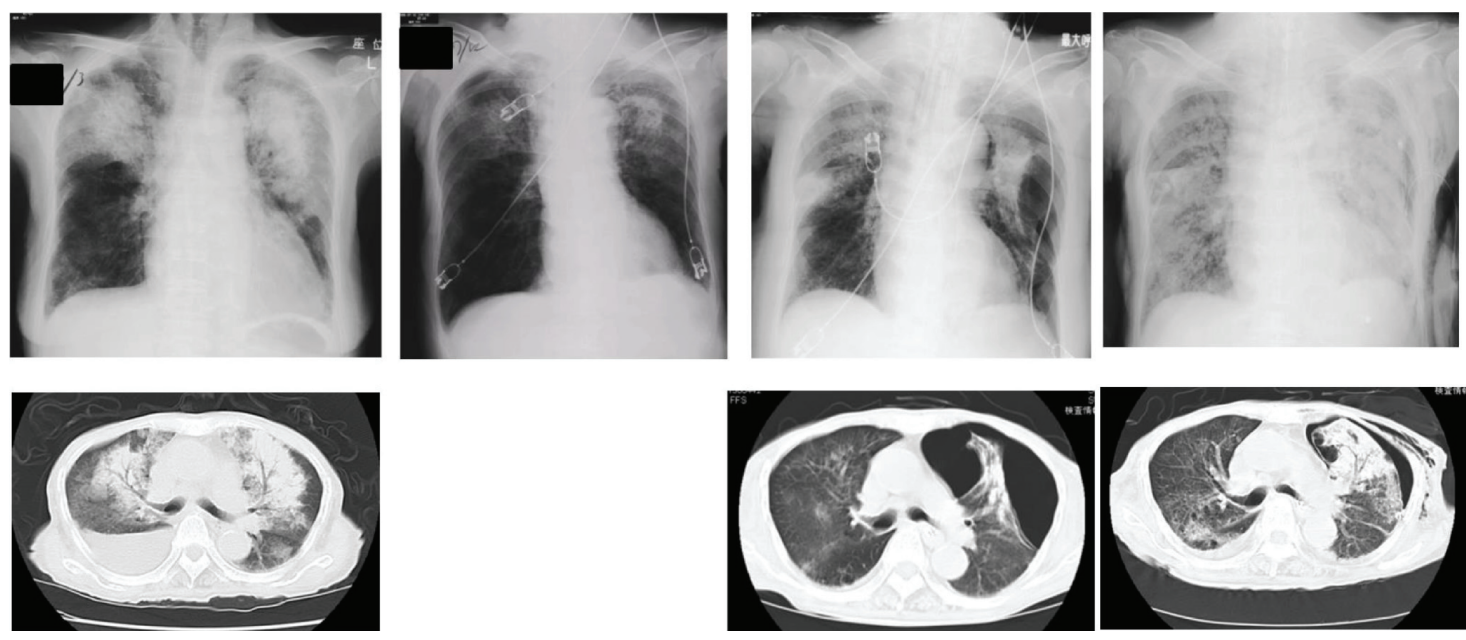

Day of

15

18

27 hospital admission

Days after hospitalization

Figure I Changes in the chest X-rays and chest computed tomography (CT) images of the patient. Chest X-rays and CT images at hospitalization and at I5, 18 , and 27 days after hospitalization are shown. 

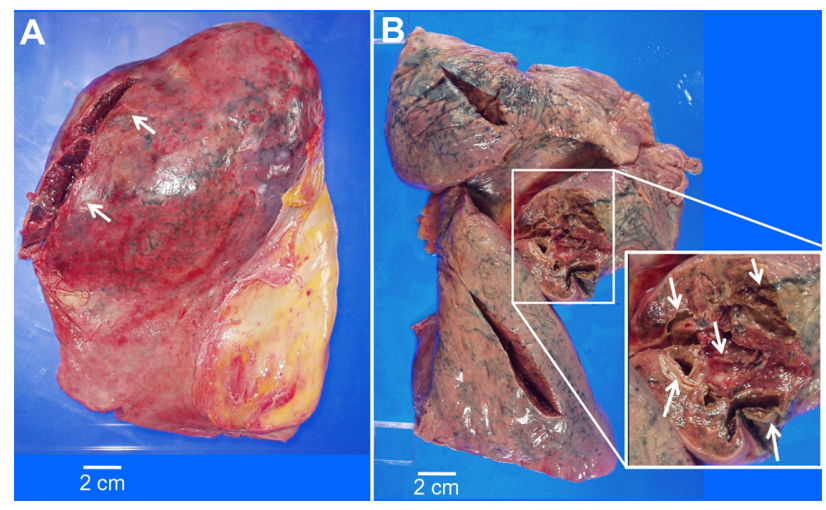

Figure 2 Macroscopic examination of the lungs. Congestion and edema were observed in the lungs bilaterally - (A) left lung, (B) right lung. The upper lobe of the left lung showed hemorrhagic necrosis and a longitudinal fissure of approximately $9 \mathrm{cms}$ long (arrows). The lower lobe of the left lung showed severe thickening of the pleura. Several cavities were observed in the middle lobe of the right lung and the inner wall of the cavities was yellow ochre in color and solid.

and necrosis of the adjacent tissue in the bilateral lung. In particular, in the upper lobe of the left lung, the hyphae existed not only around and within the blood vessels but also in the alveoli.

In the wall of the cavity in the middle lobe of the right lung, there were numerous basophilic septate hyphae with Y-shaped branching, suggesting that these hyphae were Aspergillus (Figure 4A and B). Zygomycota hyphae were observed around and within the blood vessels in the muscle layer of the left ventricle of the heart (Figure 4C and D). Many inflammatory cells had also infiltrated the cardiac muscle layer around the blood vessels. Therefore, vasculitis, thrombosis, and myocarditis

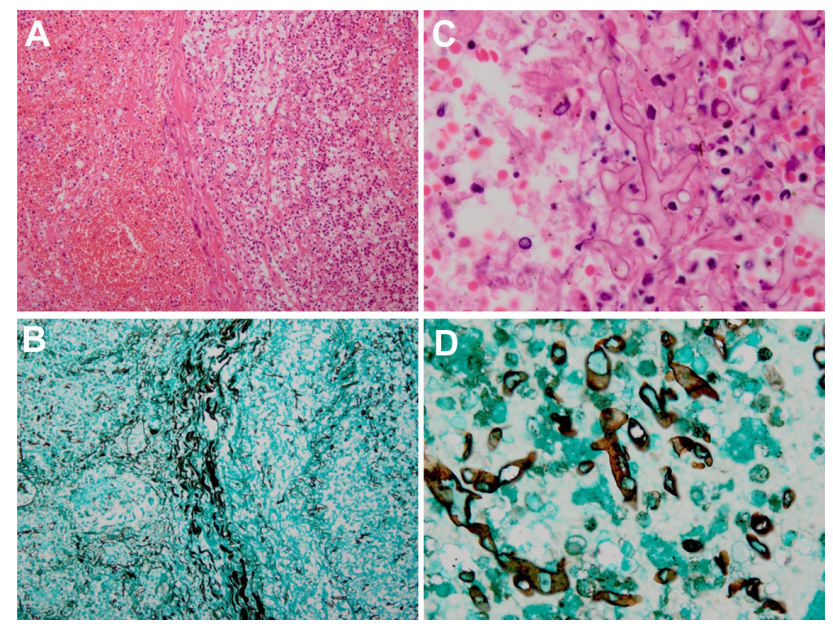

Figure 3 Microscopic examination of the lungs. The hyphae of Zygomycota were frequently observed around and within the blood vessels causing obstruction and thromboembolization of blood vessels. Non-septated, irregularly branched hyphae of Zygomycota observed used a high power field are shown in (C and $\mathbf{D}$ ). The specimens were stained with hematoxylin and eosin stain in ( $\mathbf{A}$ and $\mathbf{C})$, while those in (B and $\mathbf{D})$ were stained with Grocott's methenamine silver stain.

Notes: Magnification: (A and B), $\times 20$; (C and D), $\times 100$.
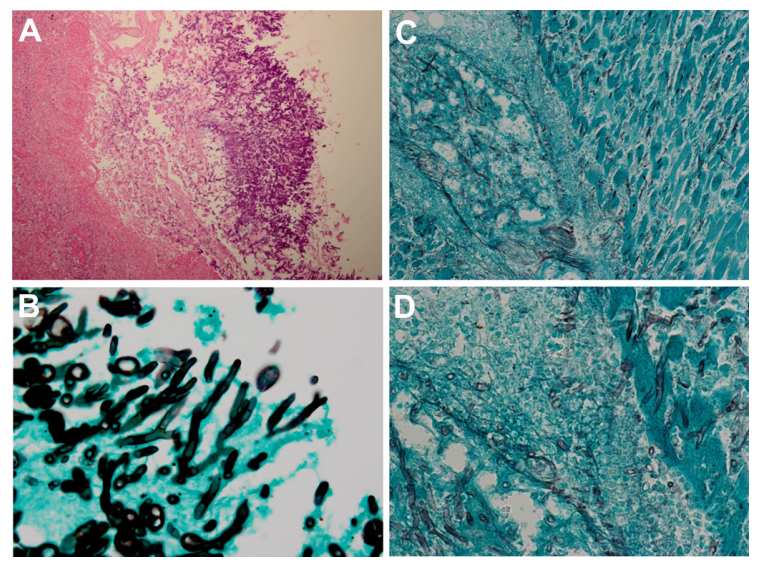

Figure 4 Microscopic examination of cavities in the middle lobe of the right lung and the heart. The hyphae of Aspergillus were observed in the wall of cavities in the middle lobe of the right lung ( $\mathbf{A}$ and $\mathbf{B})$. The hyphae show basophilic fungus bodies and Y-shaped branching. The hyphae of Zygomycota were observed around and within the blood vessels in the muscle layer of the left ventricle of the heart ( $\mathbf{C}$ and $\mathbf{D}$ ). In the blood vessels, thrombi were observed. Many inflammatory cells had infiltrated the blood vessels, suggesting that the Zygomycota infection induced vasculitis, thrombosis, and fungal myocarditis.

Notes: Magnification: (A), $\times 10$ (hematoxylin and eosin stain); (B), $\times 100$ (Grocott's methenamine silver stain); (C), $\times 20$ (Grocott's methenamine silver stain); (D), $\times 60$ (Grocott's methenamine silver stain).

caused by Zygomycota were observed in the left ventricle of the heart. Small necrotic areas of cardiac muscle were also observed. Glomerular sclerosis was observed in most of the glomeruli of both kidneys, suggesting that the kidneys were in the end stage. Chronic thyroiditis was also observed. We did not find any evidence of candidiasis.

\section{Discussion}

In this paper, we have presented an autopsy case of combined zygomycosis and aspergillosis in an immunodeficient patient that was caused by immunosuppressive therapy.

Although fungi are widespread in nature, lethal infection with fungi is very rare in healthy persons. However, infectious fungal diseases are sometimes lethal in those who are immunodeficient. Recently, a number of protocols to detect fungi have been developed. These include the detection of the specific sequence of fungal DNA, the detection of serum $\beta$-D-glucan, and enzyme-linked immunosorbent assays. However, although serum $\beta$-D-glucan increases in Candida or Aspergillus infections, it does not do so in Zygomycota. ${ }^{10}$ Enzyme-linked immunosorbent assays have also been developed for Candida and Aspergillus but not for Zygomycota. ${ }^{11}$ Therefore, it is very difficult to confirm Zygomycota infections.

Pharmaceutical agents for fungi have also been developed, including voriconazole, itraconazole, and so on. ${ }^{12}$ These medicines are easier to use than AMB due to the 
lower incidence of side effects and are therefore used prophylactically during therapy for malignancies. Zygomycota are resistant to most antifungal agents that are active against invasive aspergillosis and are increasingly encountered as breakthrough infections in patients who are receiving antifungal agents active against Candida and Aspergillus species. ${ }^{13-15}$ Only AMB deoxycholate (including lipid formulations of AMB [LAMB]) and the newer triazole, posaconazole, are active against zygomycosis. ${ }^{16,17}$ It has also been reported that delayed administration of AMB significantly increases mortality in patients with zygomycosis. ${ }^{18}$ In our case, we also used LAMB. However, LAMB was started 10 days after emergence of the patient's second dyspnea (25 days after her admission), which may have been too late to suppress the activity of the Zygomycota.

During the autopsy, hemorrhagic necrosis was seen to have developed in the left lung due to the infarction of the pulmonary blood vessels induced by Zygomycota. The left lung had become very fragile, allowing a fissure to be induced, followed by intractable pneumothorax. It has been reported that Zygomycota tend to invade the blood vessels then induce infarction; ${ }^{19}$ our case was in line with this report.

In our case, p-ANCA was positive in the serum of the patient at admission. However, we did not confirm the presence of any vasculitis except for that induced by the zygomycosis, and p-ANCA became negative 2 days before her death, suggesting that the p-ANCA-associated vasculitis had been successfully treated by plasmapheresis, steroid pulse, and azathioprine. Until 2 weeks after the patient's admission, CRP had decreased and her condition had improved. Therefore, it was thought that the zygomycosis developed 2 weeks after her admission. It has been reported that hemodialysis is associated with immunodeficiency. ${ }^{20}$ In this case, the steroid therapy, azathioprine, and plasmapheresis may have decreased the serum level of p-ANCA and improved the vasculitis, but accelerated the immunodeficiency, which may have induced the fungal infection.

\section{Conclusion}

In this report, we have described a case of combined Zygomycota and Aspergillus infection in a dialysis patient treated with immunosuppressive therapy. As previously described, ${ }^{18}$ even LAMB is not enough to suppress the activity of Zygomycota in an immunodeficient patient.

\section{Acknowledgments}

The authors thank Mr J Lee, Mr K Okano, Mr H Hongo, Mr H Egawa (Department of Anatomical Pathology, Kansai
Medical University), MrY Kinoshita, Mr K Ichibe (Surgical Pathology, Kansai Medical University - Takii Hospital), Mr H Kawakami, Ms H Ogaki, Mr K Nagaoka, Mr T Kuge (Department of Laboratory, Tyooka Hospital) for their expert technical assistance and also thank Mr Hilary Eastwick-Field and Ms K Ando (Department of Stem Cell Disorders, Kansai Medical University) for manuscript preparation.

\section{Disclosure}

The authors report no conflicts of interest in this work.

\section{References}

1. Bodey G, Bueltmann B, Duguid W, et al. Fungal infections in cancer patients: an international autopsy survey. Eur J Clin Microbiol Infect Dis. 1992;11(2):99-109.

2. Gonzalez CE, Couriel DE, Walsh TJ. Disseminated zygomycosis in a neutropenic patient: successful treatment with amphotericin B lipid complex and granulocyte colony-stimulating factor. Clin Infect Dis. 1997;24(2):192-196.

3. Oliver MR, Van Voorhis WC, Boeckh M, Mattson D, Bowden RA. Hepatic mucormycosis in a bone marrow transplant recipient who ingested naturopathic medicine. Clin Infect Dis. 1996;22(3):521-524.

4. Weitzman I, Della-Latta P, Housey G, Rebatta G. Mucor ramosissimus Samutsevitsch isolated from a thigh lesion. J Clin Microbiol. 1993;31(9):2523-2525.

5. Costa AR, Porto E, Tayah M, et al. Subcutaneous mucormycosis caused by Mucor hiemalis Wehmer f. luteus (Linnemann) Schipper 1973. Mycoses. 1990;33(5):241-246.

6. Fergie JE, Fitzwater DS, Einstein P, Leggiadro RJ. Mucor peritonitis associated with acute peritoneal dialysis. Pediatr Infect Dis J. 1992; 11(6):498-500.

7. Zabel DD. Mycotic infection with mucormycosis. Del Med J. 1997; 69(9):459-463.

8. Roden MM, Zaoutis TE, Buchanan WL, et al. Epidemiology and outcome of zygomycosis: a review of 929 reported cases. Clin Infect Dis. 2005;41(5):634-653.

9. Pagano L, Ricci P, Tonso A, et al. Mucormycosis in patients with haematological malignancies: a retrospective clinical study of 37 cases. GIMEMA Infection Program (Gruppo Italiano Malattie Ematologiche Maligne dell'Adulto). Br J Haematol. 1997;99(2):331-336.

10. Lau A, Chen S, Sleiman S, Sorrell T. Current status and future perspectives on molecular and serological methods in diagnostic mycology. Future Microbiol. 2009;4(9):1185-1222.

11. Arendrup MC, Fisher BT, Zaoutis TE. Invasive fungal infections in the paediatric and neonatal population: diagnostics and management issues. Clin Microbiol Infect. 2009;15(7):613-624.

12. Siwek GT, Dodgson KJ, de Magalhaes-Silverman M, et al. Invasive zygomycosis in hematopoietic stem cell transplant recipients receiving voriconazole prophylaxis. Clin Infect Dis. 2004;39(4):584-587.

13. Pappas PG, Alexander BD, Andes DR, et al. Invasive fungal infections among organ transplant recipients: results of the Transplant-Associated Infection Surveillance Network (TRANSNET). Clin Infect Dis. 2010; 50(8):1101-1111.

14. Imhof A, Balajee SA, Fredricks DN, Englund JA, Marr KA. Breakthrough fungal infections in stem cell transplant recipients receiving voriconazole. Clin Infect Dis. 2004;39(5):743-746.

15. Kontoyiannis DP, Lionakis MS, Lewis RE, et al. Zygomycosis in a tertiary-care cancer center in the era of Aspergillus-active antifungal therapy: a case-control observational study of 27 recent cases. $J$ Infect Dis. 2005;191(8):1350-1360.

16. Kontoyiannis DP, Lewis RE. Invasive zygomycosis: update on pathogenesis, clinical manifestations, and management. Infect Dis Clin North Am. 2006;20(3):581-607. 
17. van Burik JA, Hare RS, Solomon HF, Corrado ML, Kontoyiannis DP. Posaconazole is effective as salvage therapy in zygomycosis: a retrospective summary of 91 cases. Clin Infect Dis. 2006;42(7):e61-e65.

18. Chamilos G, Lewis RE, Kontoyiannis DP. Delaying amphotericin B-based frontline therapy significantly increases mortality among patients with hematologic malignancy who have zygomycosis. Clin Infect Dis. 2008;47(4):503-509.
19. Roden MM, Zaoutis TE, Buchanan WL, et al. Epidemiology and outcome of zygomycosis: a review of 929 reported cases. Clin Infect Dis. 2005;41(5):634-653.

20. Birkeland SA. Uremia as a state of immune deficiency. Scand $J$ Immunol. 1976;5(1-2):107-115.

International Journal of General Medicine

\section{Publish your work in this journal}

The International Journal of General Medicine is an international, peer-reviewed open-access journal that focuses on general and internal medicine, pathogenesis, epidemiology, diagnosis, monitoring and treatment protocols. The journal is characterized by the rapid reporting of reviews, original research and clinical studies across all disease areas.

\section{Dovepress}

A key focus is the elucidation of disease processes and management protocols resulting in improved outcomes for the patient.The manuscript management system is completely online and includes a very quick and fair peer-review system. Visit http://www.dovepress.com/ testimonials.php to read real quotes from published authors.

Submit your manuscript here: http://www.dovepress.com/international-journal-of-general-medicine-journal 